\title{
Need for Reappraisal of Hand-Assisted Laparoscopic Surgery for Colorectal Diseases in the Era of Desiring Small Incisions
}

\author{
Chang-Nam Kim \\ Department of Surgery, Eulji University School of Medicine, Daejeon, Korea
}

See Article on Page 125-129

Colorectal surgeons have many options for minimally invasive surgery (MIS), including standard laparoscopic surgery (SLS), single-port surgery, robotic surgery, a transanal total mesorectal excision (TME), and hand-assisted laparoscopic surgery (HALS). Traditionally, SLS is the most common type of MIS. However, nowadays, MIS using small incisions, for example, single-port surgery and transanal TME, seems to be attractive because we are living in an era when surgeons and patients desire smaller incisions. In this regard, HALS seems to be an unattractive type of surgery because a skin incision of at least $6-8 \mathrm{~cm}$ is required to insert the hand-port device. For proponents of SLS, HALS is difficult to accept because the hands rather than laparoscopic instruments are used. The penetration rate of HALS seems to be different in different countries. In northeast Asian countries, including Korea, Japan, and China, of the various types of MIS, SLS is dominant. However, among these countries, China seems to be something different. HALS seems to be relatively more popular in China than in the other two countries because China has more patients that require surgery per colorectal surgeon than the other 2 countries.

In my opinion, some reasons the penetration rate of HALS is low, especially in northeast Asian countries, are as follows: (1) Patients with high body mass index (BMI) are less common than in Western countries [1-3]. Because MIS for patients with high BMI is more technically demanding, HALS is more commonly performed in the West than in Asia. (2) Standardization of place-

Correspondence to: Chang-Nam Kim, M.D.

Department of Surgery, Eulji University Hospital, Eulji University School of Medicine, 95 Dunsanseo-ro, Seo-gu, Daejeon 35233, Korea

Tel: +82-42-259-1335, Fax: +82-42-259-1335

E-mail: kimcn7@gmail.com

(C) 2017 The Korean Society of Coloproctology

This is an open-access article distributed under the terms of the Creative Commons Attribution NonCommercial License (http://creativecommons.org/licenses/by-nc/4.0) which permits unrestricted noncommercial use, distribution, and reproduction in any medium, provided the original work is properly cited. ments of the hand-port device and laparoscopic instruments is more difficult in HALS than it is in SLS. In HALS, the concept of dominant and nondominant hand is very important. Generally, the nondominant hand should be inserted into the abdominal cavity; thus, the hand used for insertion depends on whether the surgeon is right-handed or left-handed. This is an important reason for difficulty in standardizing the procedure. (3) HALS is more comfortable for a left-handed surgeon than for a righthanded surgeon. Considering a better scope view and the usefulness of the position of the hand-port incision, the upper midline incision for a right colectomy and the lower midline incision for a left colectomy and an anterior resection are useful. (4) HALS is often misunderstood and many surgeons lack experience with the procedure. Standard laparoscopic surgeons generally have 2 options: SLS or open surgery. Even for experienced standard laparoscopic surgeons with high penetration rates of MIS, some types of MIS may be unreasonable for patients with T4 colorectal cancer, severe adhesion and ileus, and high BMI. HALS surgeons easily understand that HALS plays a key role as a type of surgery between SLS and open surgery. In a study [4] comparing 53 patients who underwent HALS and 45 patients who underwent SLS for right-sided colon cancer, the HALS group had significantly larger tumors (HALS: $6.0 \mathrm{~cm}$ vs. SLS: $3.3 \mathrm{~cm}, \mathrm{P}<0.001$ ) and significantly higher TNM stage (HALS vs. SLS: stage $0, \mathrm{n}=2$ vs. $\mathrm{n}=$ $11 ; \mathrm{I}, \mathrm{n}=5$ vs. $\mathrm{n}=15 ; \mathrm{II}, \mathrm{n}=28$ vs. $\mathrm{n}=9$; III, $\mathrm{n}=18$ vs. $\mathrm{n}=10, \mathrm{P}<$ 0.001). However, no significant differences in the 5-year overall, disease-free, and cancer-specific survival rates between the HALS and the SLS groups were reported. Based on these results, the authors of that study concluded that HALS might be an acceptable alternative to SLS for patients with high BMI and those with advanced right-sided colon cancer.

Cheong and Young [5] analyzed 121 HALS colorectal resections. Among the patients, $50.2 \%$ had high BMIs $\left(B M I \geq 25 \mathrm{~kg} / \mathrm{m}^{2}\right)$, and $52.9 \%$ had prior histories of abdominal or pelvic surgeries. The conversion rate to a laparotomy was $5.7 \%$ and was influenced by neither high BMI nor prior history of abdominal or pelvic surgeries. The authors applied HALS both to the treatments of patients with various diseases, including colorectal cancer, diverticular disease, tubulovillous adenomas, and ulcerative colitis and to di- 
verse surgeries, including high and low anterior resections, total colectomies, and total proctocolectomies. These results show that the indications of HALS are high BMI, prior history of abdominal or pelvic surgeries, and the need for a time-consuming surgery such as a total colectomy or a total proctocolectomy.

The number of obese patients in Asia is rapidly increasing. If we compare 3 papers about laparoscopic surgery for the treatment of patients with colorectal diseases in Korea, Japan, and the United States (US) [1-3], understanding the distribution of obese patients is not difficult. For a comparison of the 3 papers, patients were redefined according to the BMI category: nonobese (BMI $<25 \mathrm{~kg} /$ $\left.\mathrm{m}^{2}\right)$, overweight $\left(25 \mathrm{~kg} / \mathrm{m}^{2} \leq \mathrm{BMI}<30 \mathrm{~kg} / \mathrm{m}^{2}\right)$, and obese $(\mathrm{BMI} \geq$ $\left.30 \mathrm{~kg} / \mathrm{m}^{2}\right)$. In the Korean paper [1], of the 984 patients, 645 (65.5\%), 312 (31.7\%), and 27 (2.7\%) were classified as nonobese, overweight, and obese, respectively. All operations were performed using SLS. The obese group had a significantly higher conversion rate $(14.8 \%$ vs. $2.6 \%$ [nonobese group] and $2.9 \%$ [overweight group], $\mathrm{P}<0.001)$. In the Japanese paper [2], of the 1,194 patients, 926 (78\%), $243(20 \%)$, and 25 (2\%) were classified as nonobese, overweight, and obese, respectively. All surgeries were done using SLS. Although no statistically significant difference in conversion rate was observed, the obese group had a tendency to have a high conversion rate ( $4 \%$ vs. $0.4 \%$ [nonobese group] and $0.8 \%$ [overweight group], $\mathrm{P}=0.0605)$. In the US paper [3], of the 7,843 patients, 2,540 (32.4\%), 2,699 (34.4\%), and $2,604(33.2 \%)$ were classified as nonobese, overweight, and obese, respectively; 4,656 patients (59\%) underwent standard laparoscopic colectomy and 3,187 (41\%) underwent HALS colectomy. No data on the conversion rate were reported. These results easily show that the BMI distributions among the 3 countries are very different. Thus, one can conclude that this must be the most important reason for the low penetration rate of HALS in Asia. Colorectal surgeons in Asia should be prepared to utilize MIS for obese patients.

Personally, I use nearly all items of MIS, including SLS, HALS, hybrid surgery (combination of MIS and open surgery), singleport surgery, a retroperitoneal approach, a transanal approach, and robotic surgery including multiport and single-port plus one.
The reason I perform diverse types of MIS is simple. No one type of MIS is perfect. Each MIS has ambivalent advantageous and disadvantageous aspects. Therefore, because each patient is unique, as is each disease pattern, the most suitable MIS should vary from patient to patient. I try to adopt the type of MIS that will lead to the most successful clinical and oncologic outcomes for my patients. When I consider these outcomes, I conclude that HALS can be a powerful tool for treating both patients with high BMI, severe adhesion and ileus, and invasive cancer and those who need time-consuming surgery such as a total colectomy or a total proctocolectomy. This is the reason a reappraisal of HALS for the treatment of patients with colorectal diseases is needed in the era of desiring small incisions.

\section{CONFLICT OF INTEREST}

No potential conflict of interest relevant to this article was reported.

\section{REFERENCES}

1. Park JW, Lim SW, Choi HS, Jeong SY, Oh JH, Lim SB. The impact of obesity on outcomes of laparoscopic surgery for colorectal cancer in Asians. Surg Endosc 2010;24:1679-85.

2. Akiyoshi T, Ueno M, Fukunaga Y, Nagayama S, Fujimoto Y, Konishi T, et al. Effect of body mass index on short-term outcomes of patients undergoing laparoscopic resection for colorectal cancer: a single institution experience in Japan. Surg Laparosc Endosc Percutan Tech 2011;21:409-14.

3. Benlice C, Costedio M, Kessler H, Remzi FH, Gorgun E. Comparison of straight vs hand-assisted laparoscopic colectomy: an assessment from the NSQIP procedure-targeted cohort. Am J Surg 2016;212:406-12.

4. Bae SU, Park JS, Choi YJ, Lee MK, Cho BS, Kang YJ, et al. The role of hand-assisted laparoscopic surgery in a right hemicolectomy for right-sided colon cancer. Ann Coloproctol 2014;30:11-7.

5. Cheong JY, Young CJ. Hand-assisted laparoscopic surgery: a versatile tool for colorectal surgeons. Ann Coloproctol 2017;33:125-9. 